Jülich, HLRZ 111/93

\title{
On the equivalence between 2D Yukawa and Gross-Neveu models
}

\author{
E. Focht ${ }^{1,2}$, W. Franzki ${ }^{1,2}$, \\ J. Jersák ${ }^{1,2}$ and M.A. Stephanov ${ }^{3}$ \\ ${ }^{1}$ Institute of Theoretical Physics E, RWTH Aachen, D-52074 Aachen, Germany \\ ${ }^{2}$ HLRZ c/o KFA Jülich, , D-52425 Jülich, Germany \\ 3 Theoretical Physics, 1 Keble Rd., Oxford OX1 3NP, UK
}

\begin{abstract}
We study numerically on the lattice the 2D Yukawa model with the U(1) chiral symmetry and $N_{F}=16$ at infinite scalar field self-coupling. The scaling behaviour of the fermion mass, as the Yukawa coupling approaches zero, is analysed using the mean field method. It is found to agree with that of the Gross-Neveu model with the same symmetry and $N_{F}$. This is so even if the sign of the bare kinetic term of the scalar field is negative. This suggests that the 2D Yukawa models belong to the universality class of the Gross-Neveu models not only at weak scalar field self-coupling but also for a broad range of the bare parameters which is not accessible to the $1 / N_{F}$ expansion. New universality classes might arise at the crossover to the spin model universality class, however.
\end{abstract}

* Supported by Deutsches Bundesministerium für Forschung und Technologie, by Deutsche Forschungsgemeinschaft and by Jesus College, Oxford. 


\section{Introduction}

The 2D Gross-Neveu $\left(\mathrm{GN}_{2}\right)$ models with various global chiral symmetries [1] have been of continuous interest since it has been realized that these models are asymptotically free and that the dynamical mass generation (DMG) occurs [1, 2]. The 2D Yukawa $\left(\mathrm{Y}_{2}\right)$ models with the same symmetries have received very little attention, however, though they can potentially have very similar properties. These models are defined in the continuous Euclidean space by the action

$$
S_{\text {cont }}=\int d^{2} x\left[\sum_{\mu} \partial_{\mu} \varphi^{*} \partial^{\mu} \varphi+\frac{1}{2} m_{0}^{2}|\varphi|^{2}+\frac{\tilde{\lambda}}{4 !}|\varphi|^{4}+\bar{\psi} \not \partial \psi+\widetilde{y} \bar{\psi}\left(\operatorname{Re} \varphi+i \gamma_{P} \operatorname{Im} \varphi\right) \psi\right]
$$

where $m_{0}, \tilde{\lambda}$ and $\widetilde{y}$ are the bare scalar mass and bare dimensionful coupling constants, respectively. For definiteness we have written down the model with chiral U(1) symmetry and a quartic scalar field selfcoupling. The generalization to other symmetries and/or other selfcouplings is obvious. The fermion field $\psi$ consists of $N_{F}$ flavour components and $\varphi$ is a complex scalar field.

It was noticed long ago [3] that the Yukawa models in various dimensions reduce to the four-fermion models if the bare kinetic and selfinteraction terms for the scalar field vanish. This relationship in $4 \mathrm{~d}$ was used in the models of composite Higgs boson [4]. Such a relationship is a rather simple kinematical fact which is even more clearly seen in lattice regularized Yukawa theories [5, 6].

In this paper we present numerical results suggesting in $2 \mathrm{D}$ a much more profound relationship, namely that the $\mathrm{Y}_{2}$ field theory belongs in a broad range of parameters to one universality class, which is the class of the $\mathrm{GN}_{2}$ model with the same symmetry. Such equivalence might be indicated by the dimensionality of the coupling parameters in the $\mathrm{Y}_{2}$ models, but we are aware of no clear demonstration on such a general level. For large $N_{F}$ and small $\widetilde{y}, \tilde{\lambda}=O\left(1 / N_{F}\right)$ the equivalence can be shown in the large $N_{F}$ expansion [7]. Already fifteen years ago the equivalence between $\mathrm{Y}_{2}$ and $\mathrm{GN}_{2}$ has been suggested by Guralnik and Tamvakis [8] on the basis of a mean field (MF) analysis for a wide class of scalar selfcouplings. However, the MF method is traditionally considered not to be reliable in 2D.

Recently we have found numerical evidence that in analogy to the $\mathrm{GN}_{2}$ models the $\mathrm{Y}_{2}$ models with the Z(2) and U(1) symmetry on the lattice are asymptotically free in the Yukawa coupling even if the selfcoupling of the scalar field is arbitrarily strong [9, 10]. This property is suggested by the MF method [10] and in the effective potential approach [12] which is in spirit similar to the MF approximation. It is argued in ref. [10 that the MF method is applicable to the $\mathrm{Y}_{2}$ models because the fermions induce long range effective interaction which couples many scalar variables ferromagnetically. Such interaction can be well described by a mean field. The local selfinteractions of the scalar field (without fermions) are treated exactly or numerically. Such a version of the MF method is applicable on finite lattices and gives a good agreement with the numerical data. This allowed us to demonstrate the exponential decrease of the fermion mass with $1 / \widetilde{y}^{2}$, and thus the asymptotic freedom of the $\mathrm{Y}_{2}$ models.

The agreement between the numerical and MF methods obtained until now suggests to test numerically for various values of the coupling parameters and with improved data the MF inspired conjecture [8, 12, 10] that the $\mathrm{Y}_{2}$ and the $\mathrm{GN}_{2}$ models with the same symmetry belong to the same universality class, i.e. correspond to the same renormalized quantum field theory and have the same physical content in a broad range of the coupling parameters.

For this purpose we have investigated quantitatively the rate with which the fermion mass $a m_{F}$ in lattice units ( $a$ being the lattice constant) approaches zero as the bare Yukawa 
coupling $\widetilde{y}$ decreases. We compare this $\widetilde{y}$-dependence with the MF predictions for various fixed values of the dimensionless bare parameters $a^{2} m_{0}^{2}$ and $a^{2} \widetilde{\lambda}$. The parameters should be fixed such that the pure scalar model at $y=0$ is in the high temperature phase (symmetric phase in the $\mathrm{Z}(2)$ case, vortex phase in the $\mathrm{U}(1)$ case, etc.). The expected asymptotic scaling law in the infinite volume is

$$
a m_{F} \sim \exp \left(-\frac{1}{2 \beta_{0}} \frac{1}{g^{2}}\right),
$$

where

$$
g^{2}=\chi y^{2}
$$

$y$ is the bare dimensionless Yukawa coupling of the lattice $\mathrm{Y}_{2}$ model and $\chi$ is the susceptibility of the pure scalar model at $y=0$. For positive values of the hopping parameter $\kappa$ of the scalar field (its definition is given below) one has

$$
\begin{aligned}
\chi & =\frac{Z_{\phi}}{\left(a m_{\phi}\right)^{2}}, \\
y^{2} & =2 \kappa a^{2} \widetilde{y}^{2},
\end{aligned}
$$

$a m_{\phi}$ being the renormalized mass of the scalar boson in the high temperature phase and $Z_{\phi}$ its wave function renormalization constant.

We find numerically that in the $\mathrm{U}(1)$ model with $N_{F}=16$ the coefficient $\beta_{0}$ is within certain accuracy independent both of $a m_{\phi}$ and of the strength of the bare selfcoupling of the scalar field. This is so even if this coupling approaches infinity. The values of $\beta_{0}$ obtained from the numerical simulation are close to the value of the first coefficient of the $\beta$-function of the corresponding $\mathrm{GN}_{2}$ model. The agreement is good (within $7 \%$ ) at large $a m_{\phi}$ and is found to be even better if the sign of the bare kinetic term of the scalar field is chosen negative. On the other hand, for small $a m_{\phi}$ significant deviations from the MF prediction are observed in the range of couplings and lattice sizes we have investigated. This might indicate a crossover towards the scaling properties of the pure scalar theory.

The parameter region corresponding to the negative sign of the bare scalar kinetic term (negative $\kappa$ ) is of particular interest. Here, far outside of the scope of the perturbation expansion, the contributions from the fermion determinant to the effective kinetic term of the scalar field make the $\mathrm{Y}_{2}$ models apparently field theoretically as consistent as the $\mathrm{GN}_{2}$ models.

Further support for the conjecture of the universality of the $\mathrm{Y}_{2}$ and $\mathrm{GN}_{2}$ theories is provided by our numerical results for the ratio of the mass of the scalar fermion-antifermion bound state $B$ and of the fermion mass, $m_{B} / m_{F}$. The mass $a m_{B}$ is extracted from the two-point function of the composite operator $\bar{\psi} \psi$. The ratio is found to be independent of the chosen values of the bare parameters of the $\mathrm{Y}_{2}$ model. For $N_{F}=16$ fermions it is close to 2 and in agreement with the predicted [13, 14] value 1.99 in the $\mathrm{GN}_{2}$ model.

The outline of the paper is as follows: In the next section we define the lattice $\mathrm{Y}_{2}$ models and describe the structure of their phase diagram, as implied by the MF method and our previous numerical results. The various universality classes, suggested by this structure, are discussed. In sec. 3 we show how the universality of the $\mathrm{Y}_{2}$ models can be studied by means of the scaling behavior of the fermion mass. Here we also describe how this scaling behavior can be determined, using the MF method, on lattices of modest sizes and at rather large values of the Yukawa coupling. Section 4 presents numerical evidence obtained within the $\mathrm{U}(1)$ symmetric $\mathrm{Y}_{2}$ model with $N_{F}=16$ that the scaling behavior of the fermion mass is very close to that of the $\mathrm{GN}_{2}$ models. Some differences are noted too, however, and interpreted as a sign of a cross-over to the spin-model universality classes. In sec. 5 we further support the 
idea of equivalence between the $\mathrm{Y}_{2}$ and $\mathrm{GN}_{2}$ models by comparing the ratio of the two lowest masses. Section 6 and the appendix contains some analytic speculations about the cross-over between the $\mathrm{GN}_{2}$ and spin-model universality classes. A brief summary is presented in sec. 7 .

\section{Phase diagram of the $\mathrm{Y}_{2}$ model}

We investigate Yukawa models with $\mathrm{Z}(2)$ and $\mathrm{U}(1)$ chiral symmetry using mostly staggered fermions. The lattice actions have been chosen such that the $\mathrm{GN}_{2}$ and the spin models are their simple special cases. E.g. the action of the U(1) model for staggered fermions is:

$$
\begin{gathered}
S=S_{B}+S_{F}+S_{Y} \\
S_{B}=\sum_{x} \sum_{i}\left[-2 \kappa \sum_{\mu} \phi_{x}^{i} \phi_{x+\mu}^{i}+\phi_{x}^{i} \phi_{x}^{i}+\lambda\left(\phi_{x}^{2}-1\right)^{2}\right] \\
S_{F}=\frac{1}{2} \sum_{x, \alpha, \mu}\left(\bar{\chi}_{x}^{\alpha} \eta_{\mu x} \chi_{x+\mu}^{\alpha}-\bar{\chi}_{x+\mu}^{\alpha} \eta_{\mu x} \chi_{x}^{\alpha}\right) \\
S_{Y}=y \sum_{x, \alpha} \bar{\chi}_{x}^{\alpha} \sum_{b \in P}\left(\phi_{x-b}^{1}+i(-1)^{x_{1}+x_{2}} \phi_{x-b}^{2}\right) \chi_{x}^{\alpha}
\end{gathered}
$$

where $\phi$ is the two-component scalar field and $\chi^{\alpha}, \alpha=1, \ldots, N$, are $N$ staggered fermion fields, which describe in the continuum limit $N_{F}=2 N$ Dirac fermions. We chose the representation $\eta_{1 x}=(-1)^{x_{2}}$ and $\eta_{2 x}=1$ for the sign factors. The dimensionless bare parameters are $\kappa$, the scalar hopping parameter, $\lambda$, the scalar quartic selfcoupling and $y$, the hypercubic Yukawa coupling, which couples the scalar fields on a plaquette $P$ to the fermionic fields located in one corner of $P$. The action for the $\mathrm{Z}(2)$ model is similar, $\phi$ are then only 1-component scalar fields.

The continuum action (1.1) arises from the lattice action (2.1) if $a \rightarrow 0$ and the following rescalings are made:

$$
\begin{aligned}
\varphi & =\sqrt{2 \kappa} \phi \\
m_{0}^{2} & =(1-2 \lambda-4 \kappa) /\left(a^{2} \kappa\right) \\
\widetilde{\lambda} & =6 \lambda /\left(a^{2} \kappa^{2}\right) \\
\widetilde{y} & =y /(a \sqrt{2 \kappa})
\end{aligned}
$$

The components of $\psi$ arise from those of $\chi / \sqrt{a}$ in a complicated but standard way.

In the case $\kappa=\lambda=0$ this action describes the Gross-Neveu models on the lattice with $\phi$ being an "auxiliary" scalar field. The usual $\mathrm{GN}_{2}$ coupling constant is

$$
g=y / \sqrt{2} .
$$

The parametrization used in (2.1) makes it obvious that the $\mathrm{GN}_{2}$ models are special cases of the $\mathrm{Y}_{2}$ models. In the parametrisation usual in the continuum (1.1) the case $\kappa=\lambda=0$ corresponds to the nonperturbative limit $a^{2} m_{0}^{2}, a^{2} \widetilde{y}^{2} \rightarrow \infty, \widetilde{y}^{2} / m_{0}^{2}=g^{2}$ fixed.

The other well understood limit is $y=0$, where the action (2.1) describes the pure 2component scalar field theory and free massless fermions. In the limit $\lambda=\infty$ it is the 2D $\mathrm{XY}$ model. In the $\mathrm{Z}(2)$ case the $2 \mathrm{D}$ Ising model is obtained in this limit.

The $1 / N_{F}$ expansion usually used for the $\mathrm{GN}_{2}$ model can be applied to the $\mathrm{Y}_{2}$ models only for $\lambda=O\left(1 / N_{F}\right)$ and leads to results which are equivalent, at least in the leading $1 / N_{F}$ order, to the $\mathrm{GN}_{2}$ case [7]. In [10] we argued that the MF approximation can be used to 
describe the scaling behavior and also some finite cutoff and finite size effects in the $\mathrm{Y}_{2}$ models for any $\lambda$. The fermion determinant generates nonlocal ferromagnetic interactions between $\phi$ 's. The MF approximation is applicable because a large number of sites contribute to the mean field.

The method is briefly as follows. The effect of the fermion determinant can be described by a mean field $H(\sigma, y)$ acting on $\phi$ as an external field,

$$
H(\sigma, y)=N y^{2} \sigma \int \frac{d^{2} p}{(2 \pi)^{2}}\left(\sum_{\mu} \sin ^{2} p_{\mu}+(y \sigma)^{2}\right)^{-1},
$$

where $\sigma$ is the mean magnetization. The mean field (2.7) acts as an external field on the scalar model given by the action $S_{B}$ from (2.1) and the magnetization $\langle\phi\rangle$ is given by the selfconsistency equation

$$
\langle\phi\rangle=f(H(\langle\phi\rangle, y)),
$$

and $a m_{F}=y\langle\phi\rangle$. Here $f(H)$ is the response function of the scalar model. On a finite lattice the momentum integrals in $H(\langle\phi\rangle, y)$ are replaced by sums over lattice momenta. The resulting equation is solved numerically by recursion predicting $\langle\phi\rangle$ as a function of the Yukawa coupling $y$, the scalar couplings $\kappa, \lambda$ and the volume of the system $L^{2}$. In order to obtain the response function $f(H)$ we study the scalar model, with interactions from the pure scalar part of the action, in the external field $H$. This we can do numerically and in some special cases exactly. The mean field $H$ can then be determined selfconsistently.

We note that the logarithmically diverging nonlocality of the ferromagnetic interaction producing the mean field in (2.7) makes the DMG possible at arbitrarily weak Yukawa coupling and leads to the asymptotic freedom.

As discussed in [10] the MF approximation leads also to a better understanding of the fermion mass generation mechanism in the case of the $\mathrm{Y}_{2}$ model with continuous chiral symmetry which cannot be broken spontaneously according to the Mermin-Wagner-Coleman theorem. The main idea is that the effective interaction induced by the fermions can produce ferromagnetic ordering of scalar variables on distances $O\left(1 / m_{F}\right)$. On larger distances the ordering is destroyed by the long wavelength fluctuations (spin waves) as usual in two dimensions. The scalar field $\phi$ in a volume of a linear size $O\left(1 / m_{F}\right)$ has a nonzero magnetization $\langle\phi\rangle_{1 / m_{F}}$ which direction is drifting slowly. The mass of the fermion coupled to such a scalar field is given by $a m_{F} \simeq y\langle\phi\rangle_{1 / m_{F}}$. We checked this relation and found a good agreement in our Monte Carlo simulations. One can view this relation as a selfconsistency equation for $m_{F}$. Alternatively it can be used as a formal definition of $\langle\phi\rangle_{1 / m_{F}}$. Thus we can apply the $\mathrm{MF}$ approach to the $\mathrm{Z}(2)$ and $\mathrm{U}(1)$ models in complete analogy if we bear in mind that by $\sigma$ we mean $\langle\phi\rangle_{1 / m_{F}}$.

Figure 1 is a schematic representation of the phase diagram of the $\mathrm{Y}_{2}$ models with $\mathrm{Z}(2)$ or $\mathrm{U}(1)$ chiral symmetry. At $y=0$ we have a critical line $\kappa_{c}(\lambda)$ separating two phases of the pure scalar field theory. In the $\mathrm{Z}(2)$ case these are the high temperature, paramagnetic (PM) phase and the low temperature, ferromagnetic (FM) phase. In the $\mathrm{U}(1)$ case the corresponding phases are called vortex (VX) phase and spin wave (SW) phase, respectively, alluding to the dominant spin configurations.

There is considerable evidence provided by some exact, semiclassical and large $N_{F}$ results [1, 13, 14, 2, 15] that the $\mathrm{GN}_{2}$ models are asymptotically free and exhibit the DMG. This implies that on the line $\kappa=\lambda=0$ the systems are for arbitrarily small coupling $y$ in the broken (FM) phase for the Z(2) symmetry or in the SW phase in the U(1) case. The models have an essential singularity at $y=0$. Analytic and numerical investigations of the Yukawa models [8, 9, 12, 10] indicate that for $\kappa<\kappa_{c}(\lambda)$ the theories behave for any $\lambda$ similarly to 


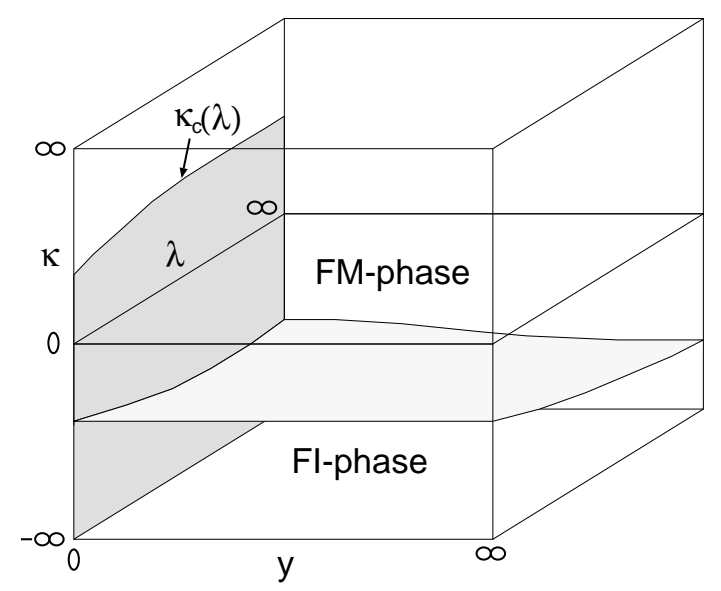

Figure 1: Sketch of the phase structure of 2 dimensional Yukawa models with hypercubic Yukawa coupling in the 3 dimensional coupling parameter space.

the $\mathrm{GN}_{2}$ models. They are asymptotically free. At arbitrarily small $y$ the models are in the FM or SW phase for the $\mathrm{Z}(2)$ or $\mathrm{U}(1)$ case, respectively. The surface $\kappa<\kappa_{c}(\lambda), y=0$ is a critical surface at which the masses of the $\mathrm{GN}_{2}$ model vanish, if expressed in lattice units. One can perform the continuum limit approaching any point of this surface. For some lattice discretizations of the $\mathrm{GN}_{2}$ or Yukawa models on the PM or VX phases can appear at large $y$ [16, 9]. This is a lattice artifact, which can be avoided by using the hypercubic Yukawa coupling.

The scalar part of the action (2.1) has the so-called staggered symmetry

$$
\phi_{x} \rightarrow(-1)^{x_{1}+x_{2}} \phi_{x}=: \phi_{x}^{s t}, \kappa \rightarrow-\kappa
$$

In the scalar models this symmetry implies a phase transition line at $\kappa=-\kappa_{c}(\lambda)$ which separates the PM and the antiferromagnetic phase in the $\mathrm{Z}(2)$ model and the VX phase from the staggered spin wave phase in the $\mathrm{U}(1)$ case.

The staggered scalar field $\phi^{\text {st }}$ does not couple to the fermions through the hypercubic Yukawa coupling (2.4). Therefore we expect the phase transition at $\kappa=-\kappa_{c}(\lambda)$ to occur also for $y>0$ and to depend only weakly on $y$. It forms a critical sheet which separates in the $\mathrm{Z}(2)$ case the FM from the FI (ferrimagnetic) phase (see fig. 1). We have checked the existence of the FI phase in the case of the Z(2) model numerically. A similar critical sheet is expected also in the $\mathrm{U}(1)$ model.

Though we do not know whether the critical sheet $y=0, \kappa \leq \kappa_{c}(\lambda)$ extends to $\kappa=-\infty$ or not, we see no reason why it shouldn't. The phase transition around $\kappa \simeq-\kappa_{c}(\lambda)$ does not influence $a m_{F}$ which seems to scale with $y \rightarrow 0$ also below this sheet.

The described phase structure suggests the existence of several universality classes in the $\mathrm{Y}_{2}$ models. They depend on the position of the critical point where one performs the continuum limit and, as we shall see, in some cases probably also on the way how this point is approached.

When one performs the continuum limit on the critical line $\kappa_{c}(\lambda)(\lambda>0)$ and approaches it within the plane $y=0$, in the $\mathrm{Z}(2)$ case the theory belongs to the Ising universality class. In the $\mathrm{U}(1)$ case the transition is Kosterlitz-Thouless-like, the theory belongs to the universality class of the 2D XY model. At $\lambda=0$ and $\kappa=1 / 4$ there is the Gaussian fixed point. When approaching this point from smaller $\kappa$ at $y=0$ we have a theory with free noninteracting scalars and free massless fermions. The continuum limit towards the line $\kappa=\kappa_{c}(\lambda)$ taken from the $y>0$ region is rather complex and will be discussed in section 6 . 
Approaching the critical surface $y=0, \kappa<\kappa_{c}(\lambda)$, leads to an asymptotically free theory [8, 9, 12, 10]. But it is not a priori clear whether this theory is for any $\lambda>0$ and $\kappa<\kappa_{c}(\lambda)$ in the same universality class as the $\mathrm{GN}_{2}$ model at $\lambda=\kappa=0$. At least the spectrum is expected to be similar, with fermions and fermionic bound states scaling while the masses of the states in the $\phi^{4}$ theories, in particular $a m_{\phi}$, remain nonzero and decouple in such a continuum limit. The discussion of this limit is the main aim of the next two sections of this paper. We shall concentrate on two aspects of universality: the question whether the theories scale in the same way when one approaches the critical surface at different $(\kappa, \lambda)$ points. More precisely, whether the first coefficient of the $\beta$ function, which can be interpreted as a critical exponent, is the same. We also examine the ratio of the first two masses in the spectrum of the $\mathrm{Y}_{2}$ model and compare it with that of the $\mathrm{GN}_{2}$ model.

We note that the structure of the phase diagram and the continuation of the critical surface at $y=0$ to negative $\kappa$ suggests to consider also $\kappa<0$, though the rescalings (2.5) are not well defined. Even if negative $\kappa$ corresponds to the bare scalar kinetic term with wrong sign, the continuum $\mathrm{Y}_{2}$ theories can be physically sensible at $\kappa<0$. A strong indication for such a phenomenon has been found numerically in the 4D Yukawa theories on the lattice for sufficiently strong Yukawa coupling [17]. Thus we shall study the scaling behavior at negative $\kappa$ also.

At the critical surface around $\kappa \simeq-\kappa_{c}(\lambda), y>0$, the fermion mass does not scale and fermions would decouple in the corresponding continuum limit.

\section{Scaling behaviour of $a m_{F}$ and its analysis on finite lattices}

The asymptotic scaling law for the GN model (1 loop) is

$$
a m_{F} \sim \exp \left(-\frac{1}{2 \beta_{0}} \frac{1}{g^{2}}\right) .
$$

We note that the correct values of $\beta_{0}$ obtained in perturbation theory are

$$
\beta_{0}=\frac{N_{F}-1}{2 \pi} \quad(\mathrm{Z}(2)), \quad \beta_{0}=\frac{N_{F}}{2 \pi} \quad(\mathrm{U}(1))
$$

The MF considerations [10, 18] and the study of the effective potential [12] predict that the fermion masses in the more general case of the $\mathrm{Y}_{2}$ models behave according to the scaling law ([1.2). Here $\chi=\chi(\kappa, \lambda)$ is the susceptibility of the pure scalar model described by the part $S_{B}$ of the action (2.1). For $0<\kappa<\kappa_{c}(\lambda)$ it is given by (1.4), but the scaling law (1.2) holds also for $\kappa \leq 0$. Indeed, $\chi$ is finite for such $\kappa$ values. In particular,

$$
\chi(\kappa, 0)=\frac{1-4 \kappa}{2 \kappa} .
$$

The predicted value $\beta_{0}=N_{F} / 2 \pi$ does not depend on $\kappa$ and $\lambda$ and thus coincides with the value of the first coefficient of the $\beta$ function of the $\mathrm{GN}_{2}$ model in eq. (3.1), as predicted by the MF approximation in that case. In the $\mathrm{U}(1)$ case the MF approximation gives $\beta_{0}$ correctly. We note that the scaling law (3.1) of the $\mathrm{GN}_{2}$ model agrees with (1.2) because of eq. (2.6) and $\chi(0,0)=1 / 2$.

As mentioned in the previous section we concentrate on the question whether the continuum limit taken at the critical surface $y=0, \kappa<\kappa_{c}(\lambda)$ is everywhere Gross-Neveu like. 


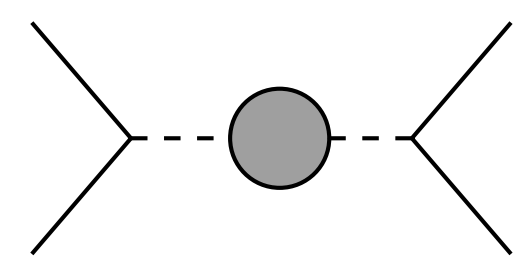

Figure 2: The 4 point function in a Yukawa theory in the lowest order in $y$ and the scalar selfinteraction fully included.

Therefore we want to compare the scaling behaviour of the fermion mass $a m_{F}$ for $y \rightarrow 0$ at various fixed $\kappa$ and $\lambda$. In order to make such a comparison it is necessary first to compare the strength of the Yukawa coupling itself. The interacting scalar field namely modifies the effective strength of the Yukawa interaction already on the fermionic tree level. This is obvious when the fermion four-point function $G_{F}^{(4)}$ is considered in the lowest order in y, namely $\mathrm{O}\left(y^{2}\right)$, but with the scalar selfinteraction fully included, as shown in Fig. 2. At zero momentum we obtain

$$
G_{F}^{(4)}\left(p_{i}=0\right)=y^{2} G_{\phi}^{(2)}(p=0)=y^{2} \chi .
$$

Thus we see that the scaling behaviour should be compared with respect to the effective strength of the Yukawa coupling

$$
g^{2}=\chi y^{2}
$$

(We thank F. Niedermayer for an elucidating discussion on this point.) Therefore the scaling behavior of $a m_{F}$ for various fixed $\kappa, \lambda$ values is interpreted as the same if the numerically determined coefficient $\beta_{0}$ in the scaling law (1.2) is the same. Its consistency with the values (3.2) is then an indication that the $\mathrm{Y}_{2}$ models belong for those $\kappa, \lambda$ values to the $\mathrm{GN}_{2}$ universality class.

The aim of our numerical investigations is thus to check whether the scaling law (1.2) is consistent with the data. However, eq. (1.2) is valid only in the infinite volume limit at very small $y$. In ref. [10] we described how the MF method can make predictions about the fermion masses on finite lattices.

These MF predictions allow us to estimate how Yukawa models approach the asymptotic scaling. In fig. 3 we have plotted the fermion mass $a m_{F}$ obtained by solving eq. (2.8) for $\kappa=\lambda=0$ on lattices with $L=16,32,64$ and 256. The onset of asymptotic scaling represented by the straight line is around $1 / g^{2} \approx 20$, i.e. it can be seen only on very large lattices $(L \gtrsim 200)$.

Figure 1 shows the MF prediction for $a m_{F}$ at $\lambda=\infty$ and $\kappa=-0.2$. The picture is similar to the $\mathrm{GN}_{2}$ case, but an indication of the same asymptotic scaling seems to require even larger lattices. For other $\kappa$ at $\lambda=\infty$ the situation is analogous.

The MF analysis thus suggests such a slow approach to the asymptotic scaling that we have no chance to achieve it in numerical simulations. Too small $y$ and, correspondingly, too large lattices would be needed. The strategy we adopt in this situation is the following: we compare the numerical data for $a m_{F}$ with the MF predictions for finite volumes and correspondingly large $y$. If we find an agreement within a reasonable accuracy margin, we conclude that the MF method works well and that its asymptotic predictions are thus correct. In other words, the MF analysis of the data is the theoretical means for extrapolation to large $1 / g^{2}$ and $L$ of the data obtained numerically at moderate values of these parameters.

To compare numerical data with the MF predictions we introduced an ad-hoc correction $r$ to be determined in fits to the data. It's role is to take into account eventual deviations from the mean field approximation and to quantify the (dis)agreement between data and 


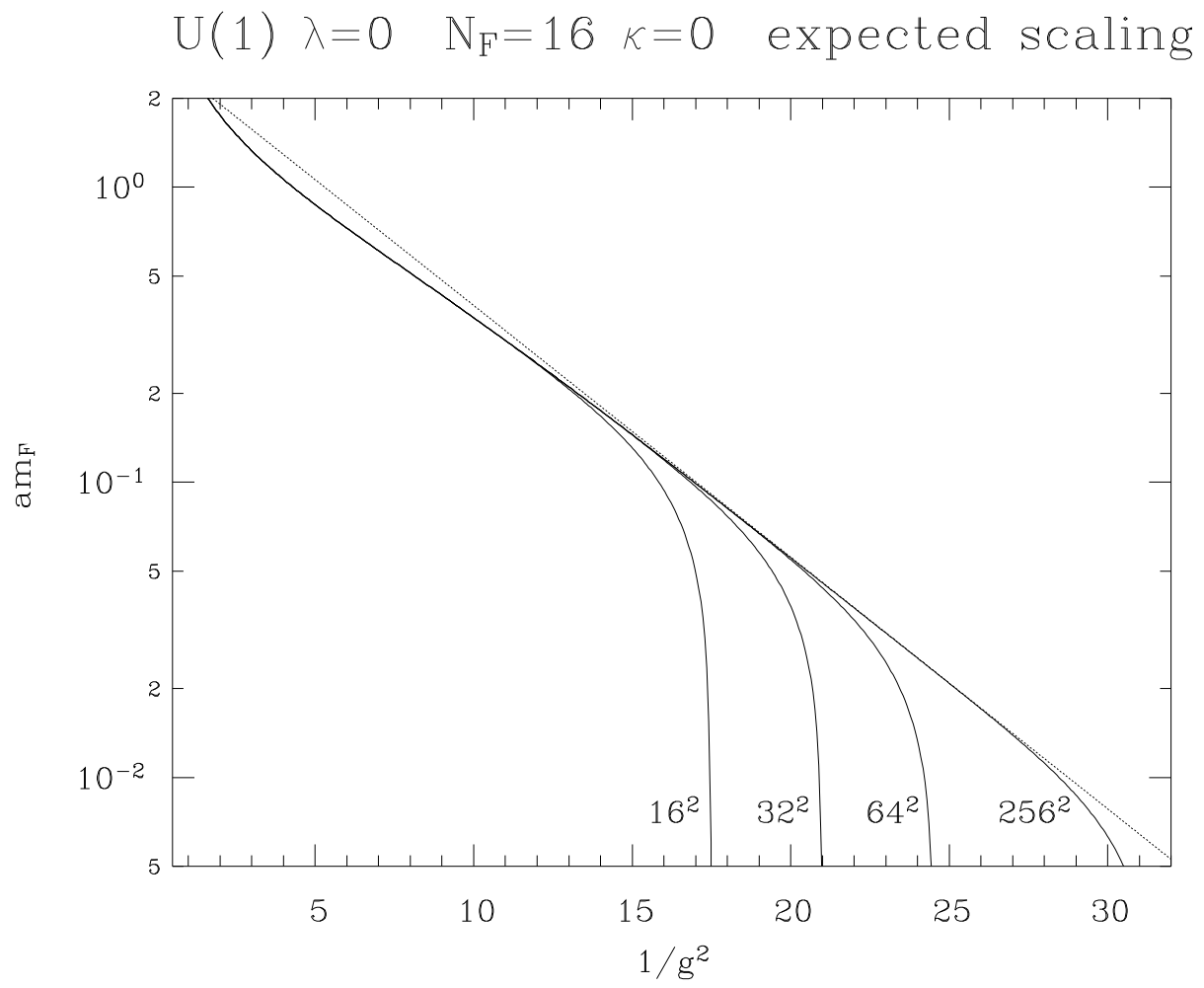

Figure 3: Mean field approximation prediction for the fermion mass in the GN case, plotted logarithmically against $1 / g^{2}=1 /\left(\chi y^{2}\right)$. The slope of the straight line corresponds to the asymptotic scaling, eq. (3.1).

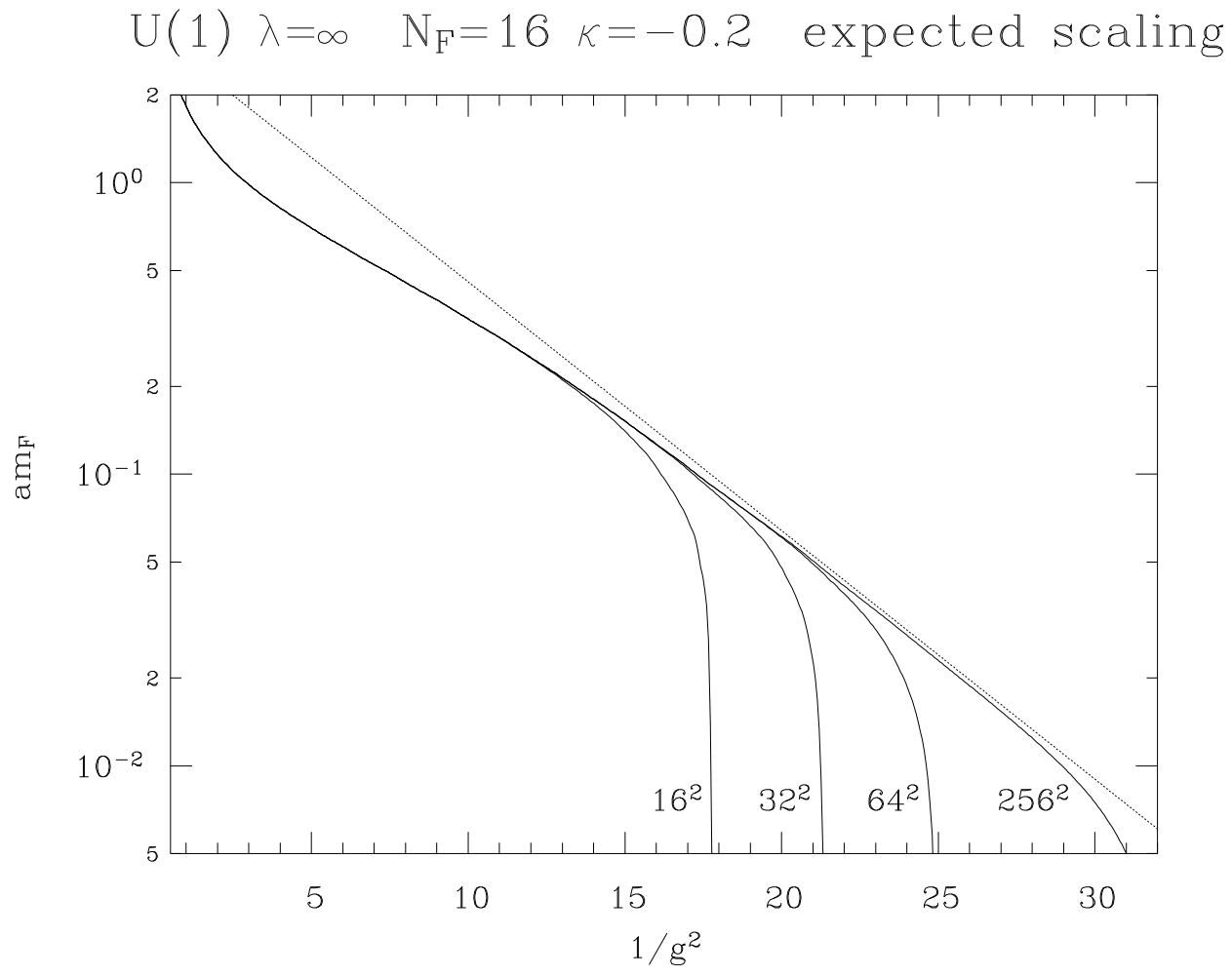

Figure 4: Same as fig. B, now for $\lambda=\infty, \kappa=-0.2$. 


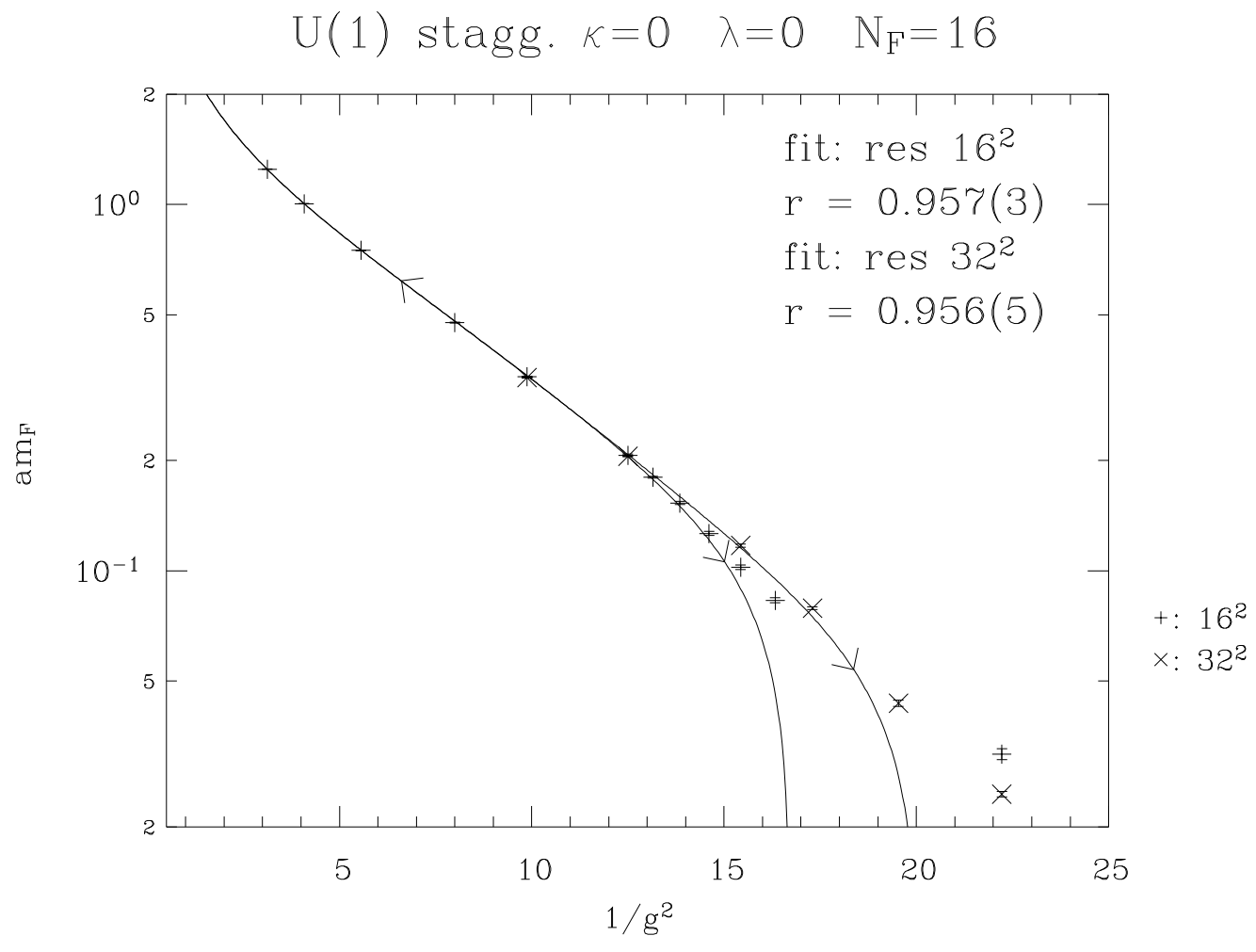

Figure 5: Numerical data for fermion mass $m_{F}$ on finite lattices in the U(1) $\mathrm{Y}_{2}$ model plotted against $1 / g^{2}$, where $g$ is the effective Yukawa coupling $g=\sqrt{\chi} y$.

the prediction. It is introduced in the following way: instead of $a m_{F}=y\langle\phi\rangle$ we use in eqs. (2.7), (2.8) $a m_{F}=r y\langle\phi\rangle$ and fit for the parameter $r$. In this MF motivated Ansatz the asymptotic scaling would have a different form, namely

$$
a m_{F} \sim e^{-\frac{1}{2 \beta_{0} r} \frac{1}{g^{2}}}
$$

However, on lattices much smaller than the sizes at which the asymptotic behavior sets on, $r$ parametrizes the difference between the MF prediction of the approach to the asymptotic scaling and the true one. So we expect $r \rightarrow 1$ as $L \rightarrow \infty$ and $y \rightarrow 0$. The result $r=1$ would mean perfect agreement between the data and the MF prediction for the coefficient $\beta_{0}$ in the asymptotic scaling behavior in eq. (1.2), which of course cannot be expected on finite lattices.

\section{Observed $y$ dependence of $a m_{F}$}

In order to verify the hypothesis that $\mathrm{GN}$ and $\mathrm{Y}_{2}$ models are in the same universality class as $y \rightarrow 0$ for $\kappa<\kappa_{c}(\lambda)$ we have studied the $y$-dependence of $a m_{F}$ in the U(1) model with $N_{F}=16$. The numerical data have been collected using staggered fermions on lattices of the sizes $L=16,32,64$ at many $y$ points for five pairs of fixed $\kappa$ and $\lambda$ values (see table 1 ). Using the hybrid Monte Carlo algorithm, the fermion propagators have been measured in coordinate and in momentum space and have been fitted with the free fermion Ansatz. The results for $a m_{F}$ are very stable and the errors so small that the $y$-dependence of this mass clearly shows deviations from the MF predictions on finite lattices.

The pair $\kappa=\lambda=0$ (the $\mathrm{GN}_{2}$ model, which is the best understood case) has been chosen to estimate the quality of the MF description of the data. For this purpose we have 


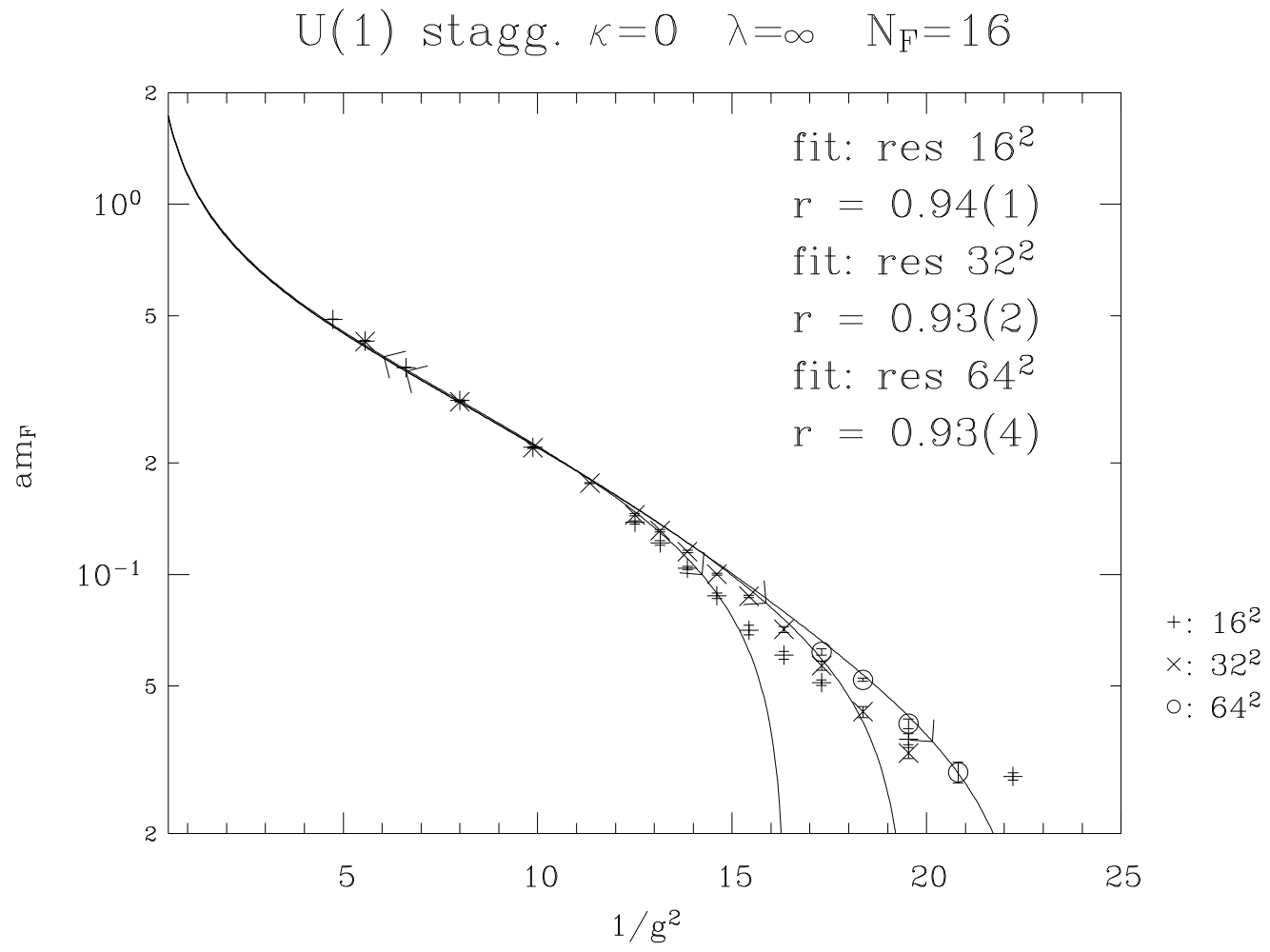

Figure 6: Same as fig. 5, now for $\lambda=\infty$ and $\kappa=0$.

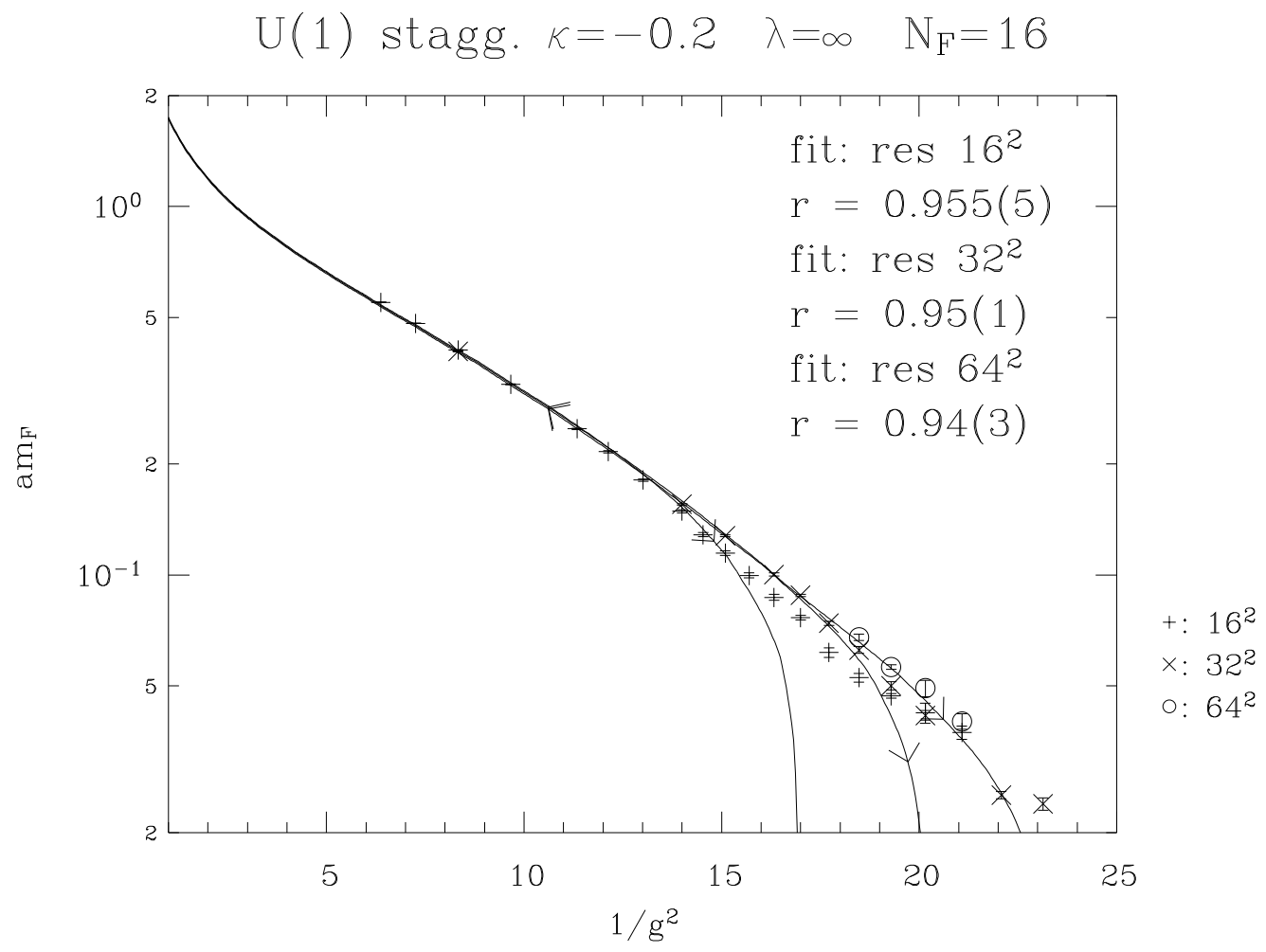

Figure 7: Same as fig. 5, for $\lambda=\infty$ and $\kappa=-0.2$. 


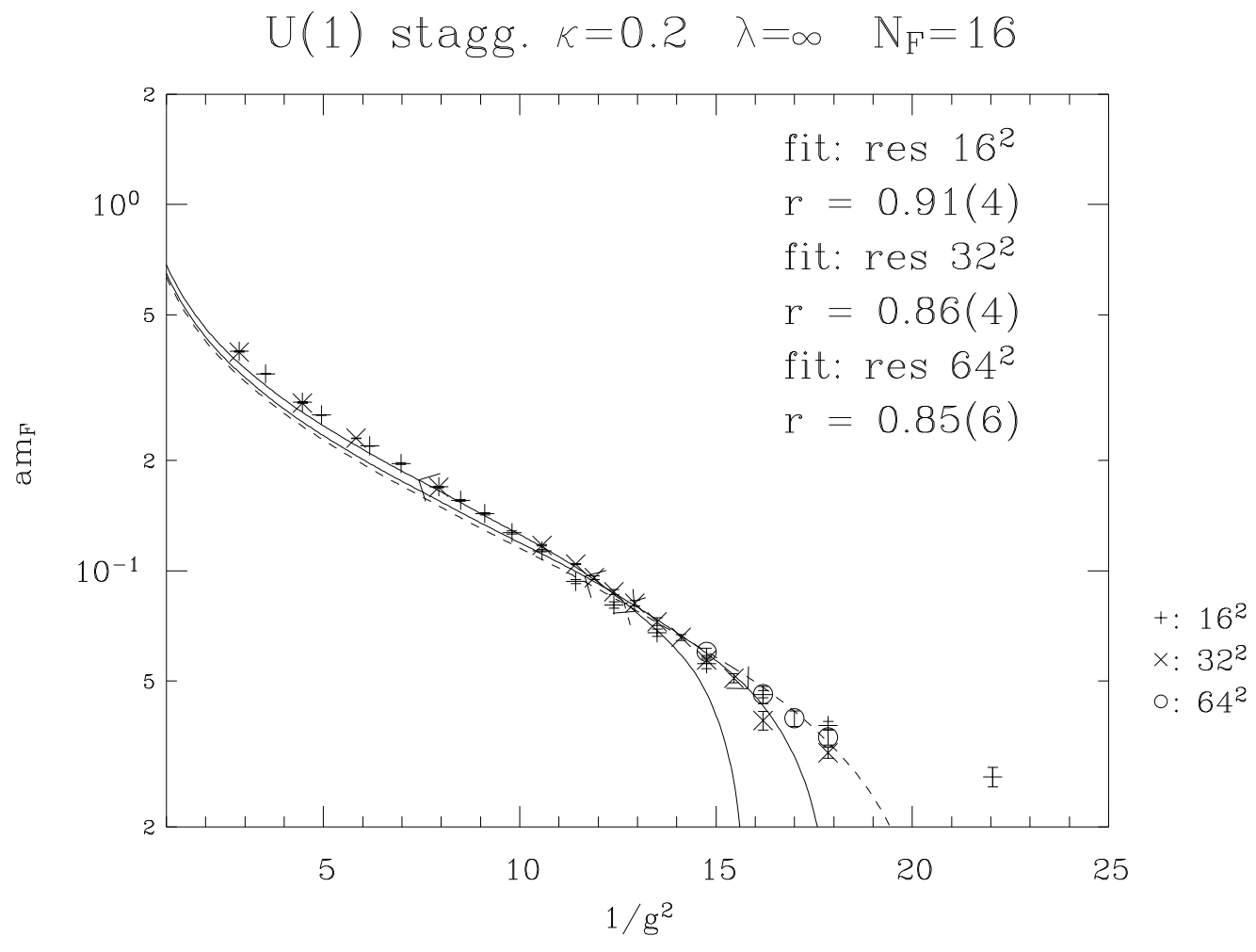

Figure 8: Same as fig. 5, for $\lambda=\infty$ and $\kappa=0.2$.

\begin{tabular}{|cc|ccc|}
\hline$\kappa$ & $\lambda$ & $16^{2}$ & $32^{2}$ & $64^{2}$ \\
\hline 0 & 0 & $0.957(3)$ & $0.956(5)$ & \\
-0.4 & $\infty$ & $0.971(5)$ & $0.970(7)$ & \\
-0.2 & $\infty$ & $0.955(5)$ & $0.95(1)$ & $0.94(3)$ \\
0 & $\infty$ & $0.94(1)$ & $0.93(2)$ & $0.93(4)$ \\
0.2 & $\infty$ & $0.91(4)$ & $0.86(4)$ & $0.85(6)$ \\
\hline
\end{tabular}

Table 1: Values of the fit parameter $r$ in the U(1) $\mathrm{Y}_{2}$ model with $N_{F}=16$ on $16^{2}, 32^{2}$ and $64^{2}$ lattices.

determined the value of the parameter $r$ by a fit to the data as described at the end of the preceding section. The fit is shown in fig. 5 and we find that $r$ has the same values for both lattice sizes $L=16,32$ we have used in this case and deviates from 1 by $4.5 \%$. Having chosen rather large $N_{F}=16$ we can be sure that the $\mathrm{GN}_{2}$ model scales according to eq. (1.2), as predicted by the large $N_{F}$ expansion. So the deviations of $r$ from 1 of this magnitude should not be interpreted as a signal for a scaling behavior different from (1.2), but rather as an estimate of the accuracy within which the MF method works on finite lattices.

In the figs. 6, 7 and 8 we present numerical results of the hybrid $\mathrm{MC}$ simulation of the $\mathrm{Y}_{2}$ model for $\lambda=\infty$ and $\kappa=0,-0.2$ and +0.2 , respectively. The results for the fit parameter $r$ are shown in the table 1. We have concentrated on $\lambda=\infty$ because this value is the opposite extreme to the case $\lambda=O\left(1 / N_{F}\right)$ when (1.2) follows from the $1 / N_{F}$ expansion. Thus an agreement with (1.2) at $\lambda=\infty$ allows to expect that a similar agreement can be found for any intermediate value of $\lambda$.

The agreement is indeed found for $\kappa \leq 0$. At $\kappa=0$ (fig. 6) we have $r \approx 0.93$. The fits are good and although the deviation of $r$ from 1 is larger than in the GN case (fig. 5), it is 
still similar to that case. The fit in fig. 7 is done for data at $\kappa=-0.2$ and the results for $r$ are the same as in the GN model (within errors). For $\kappa=-0.4$ the value of $r$ is even nearer to 1 than in the GN case (tab. 1, figure not included). There is a clear sign, that $\beta_{0}$ is the same for $\kappa=\lambda=0$ and $\kappa \leq 0, \lambda=\infty$.

At $\kappa=0.2, \lambda=\infty$ the fit in fig. 8 shows considerable deviation of $r$ from 1 , however. The quality of the fit is also poor, i.e. the data cannot be described by the MF Ansatz in spite of the presence of the $L$-dependent parameter $r$. We suppose that this is an effect of the crossover region to the other universality classes at $\kappa=\kappa_{c}(\lambda)$ (discussed in section 6 ).

The presented results for the $y$-dependence of $a m_{F}$ are only a part of the data for $a m_{F}$ we have accumulated during our studies of the $\mathrm{Y}_{2}$ models. We have investigated also the $\mathrm{U}(1)$ symmetric lattice $\mathrm{Y}_{2}$ model with naive fermions [9, 10] and for $\lambda=0$ and $\lambda=0.5$ performed also simulations of the $\mathrm{Z}(2)$ model with various $N_{F}$. Though less detailed, these results are consistent with those presented here. Put together, our results strongly suggest that, as $y \rightarrow 0$ at fixed $\lambda \geq 0$ and $\kappa$ satisfying $-\kappa_{c}(\lambda)<\kappa<\kappa_{c}(\lambda)$, the fermion mass $a_{F}$ behaves according to the scaling law (1.2). We interpret this as a piece of evidence that the $\mathrm{Y}_{2}$ models belong in the indicated region of $\lambda, \kappa$ parameters to the same universality class as the $\mathrm{GN}_{2}$ models with the same $N_{F}$ and chiral symmetry.

We note that neither the present data nor the MF method of their analysis allow to determine the values of the mass gap to compare with the exact result [15]. For this purpose either the data in the asymptotic scaling region or a much better analysis of finite cut-off effects would be needed.

\section{The mass of the $\bar{\chi} \chi$ state}

Needless to say, more observables should be compared if the equivalence of various field theoretical models is under discussion. Besides critical exponents, the simplest universal quantities are the ratios of masses. A comparison of the spectrum can give valuable hints about the universality classes of similar theories.

Early analytic investigations [13, 14] have revealed a rich spectrum in the $\mathrm{GN}_{2}$ model. The mass of the first excited state, which is interpreted as a fermion-antifermion bound state, is

$$
m_{B}=2 m_{F} \cos \left(\frac{\pi}{2\left(N_{F}-1\right)}\right),
$$

followed by other fermion-antifermion bound states and multifermion bound states.

We could determine the mass $a m_{B}$ in the lattice units. We followed the methods of ref. [19] and measured the time-dependent correlation function

$$
C_{B}\left(x_{2}\right)=\left\langle\frac{1}{L} \sum_{x_{1}}\left|M_{0 x}^{-1}\right|^{2}\right\rangle,
$$

where $x_{1}$ is the Euclidean time, $x_{2}$ the space coordinate and $M_{0 x}^{-1}$ the inverted fermion matrix. The function $C_{B}\left(x_{2}\right)$ is the correlator $\langle\bar{\chi} \chi(0) \bar{\chi} \chi(x)\rangle$ at zero spatial momentum. The operator $\bar{\chi} \chi(x)$ is local and therefore very easy to measure, but has small overlap $Z_{B}$ with the bound state we are actually looking for. One can write:

$$
C_{B}(t)=C_{F F}(t)+Z_{B}\left(e^{-a m_{B} t}+e^{-a m_{B}(T-t)}\right)+\tilde{Z}_{B}(-1)^{t}\left(e^{-a \tilde{m}_{B} t}+e^{-a \tilde{m}_{B}(T-t)}\right) .
$$

$C_{F F}$ is the free fermion-antifermion cut contribution

$$
C_{F F}(t)=\frac{1}{L} \sum_{x_{1}} G_{F}^{(2)}(0, x) G_{F}^{(2)}(0, x) .
$$




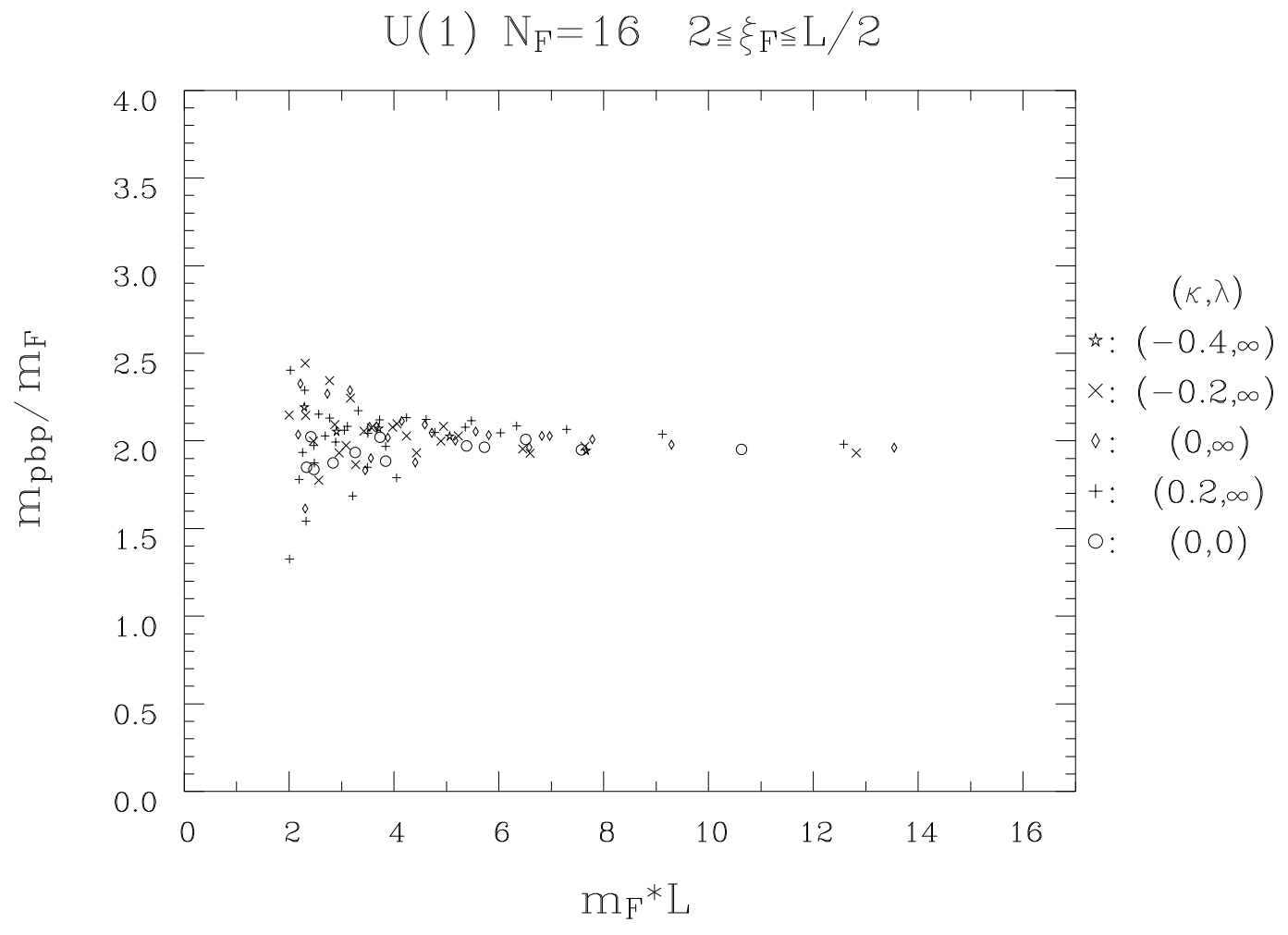

Figure 9: The ratio $m_{B} / m_{F}$ plotted vs. $a m_{F} L$. The results agree for all investigated $\kappa, \lambda$ pairs as long as the physical size of the lattice is sufficiently large, $a m_{F} L>4$.

$G_{F}^{(2)}(0, x)$ is the free fermion propagator, the corresponding $m_{F}$ and $Z_{F}$ are determined by fitting the measured fermion propagator $\langle\bar{\chi}(0) \chi(x)\rangle$.

The mass $a m_{B}$ has been obtained at nearly all simulation points. The results for those points, at which

$$
2 \leq \frac{1}{a m_{F}} \leq \frac{L}{2}
$$

are plotted in fig. 9. The first inequality in (5.5) selects points without significant lattice artifacts, whereas the second one cuts off points with too large finite size effects. In fig. 9 we display two physical, dimensionless quantities: the ratio $m_{B} / m_{F}$ and $a m_{F} L$, the size of the system in the units of the fermionic correlation length.

The finite size effects increase as $a m_{F} L$ decreases, and these effects are not expected to be universal. But we see in fig. 9 that at least for $a m_{F} L>4$, all the data are close to the expected value $m_{B} / m_{F}=1.99$. This agreement favours the conclusion that for all shown $(\kappa, \lambda)$ pairs, including the GN limit, the theory is in the same universality class.

The large uncertainties introduced by the inexact measurement of $a m_{B}$ do not allow to draw a definite conclusion, however. The cut contribution $C_{F F}(t)$ in eq. (5.3) is large: about $95 \%$. This makes the determination of $m_{B}$ not very reliable. We checked, however, that the fits for $C_{B}(t)$ with only the cut contribution are significantly worse than those with the full expression (5.3). Nevertheless, we cannot exclude the possibility that the result for the mass $m_{B} \simeq 2 m_{F}$ is an artefact of the contribution of the fermion-antifermion threshold to $C_{B}(t)$. In any case our results show that the mass of the lightest fermion-antifermion bound state is not significantly lower than $2 m_{F}$, otherwise the mass $a m_{B}$ would be easily measurable. This still supports to some extent the universality hypothesis. 


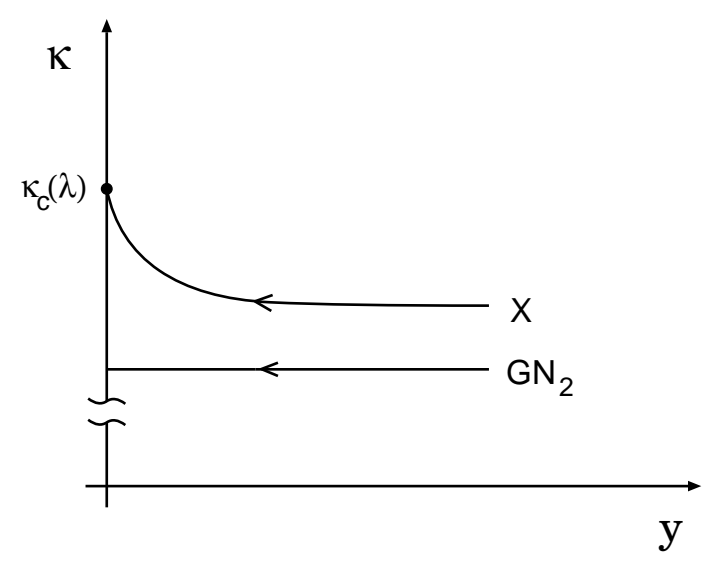

Figure 10: Two types of continuum limits from the $y>0$ in the vicinity of the line $\kappa=\kappa_{c}(\lambda)$.

\section{Transition between the Gross-Neveu and spin model universality classes}

We have tentatively concluded that the $\mathrm{Y}_{2}$ models are equivalent to the $\mathrm{GN}_{2}$ models in the continuum limit $y \rightarrow 0$ when $\kappa$ is fixed at some $\kappa<\kappa_{c}(\lambda)$. This still allows that something new and interesting happens in the limit when $y \rightarrow 0$ and $\kappa \rightarrow \kappa_{c}(\lambda)$ simultaneously. If so, this would justify an independent existence of the $\mathrm{Y}_{2}$ models as field theories with physical content different from the $\mathrm{GN}_{2}$ universality class.

A hint that this could be the case is provided by the boson spectrum considerations in the vicinity of the line $\kappa=\kappa_{c}(\lambda)$ at $y=0$. In fig. 10 we indicate the situation at some fixed $\lambda$. The surface $y=0, \kappa \leq \kappa_{c}(\lambda)$ corresponds to the high temperature phase of the scalar field theory which describes massive scalar particles of mass $m_{\phi}$. The continuum limit of this theory is obtained as $\kappa \rightarrow \kappa_{c}(\lambda)_{-}$, where $a m_{\phi} \rightarrow 0$. The universality class for $\lambda>0$ is that of the 2D spin models with the same global symmetry as the scalar field theory. The points $\kappa<\kappa_{c}(\lambda)$ are regular points of the scalar field theory, i.e. $a m_{\phi}>0$. Thus the end point of the line $G N_{2}$ is a regular point and when the continuum limit $a m_{F} \rightarrow 0$ is taken along this line, the ratio $m_{\phi} / m_{F}$ becomes infinite. The scalar theory "decouples" and the continuum $\mathrm{GN}_{2}$ model results.

The situation is different along certain lines of the type $X$ shown in fig. 10. One can imagine a fine-tuned approach towards the line $\kappa=\kappa_{c}(\lambda)$ at $y=0$ along which

$$
a m_{\phi}, a m_{F} \rightarrow 0, \frac{m_{\phi}}{m_{F}} \rightarrow \alpha
$$

with some arbitrary constant $\alpha$. Assuming applicability of the MF approximation, which can hold at least for large $\alpha$ and not very close to the point $\kappa=\kappa_{c}$, one can even calculate the corresponding paths (see Appendix).

Thus the existence of a family of continuum field theories is indicated, labeled by the ratio $\alpha=m_{\phi} / m_{F}$. Its spectrum might consist roughly of the same states as the $\mathrm{GN}_{2}$ spectrum plus the spectrum of the scalar field theory in the high temperature phase. We see no reason why these two "sectors" would not interact with each other, so that these field theories might be of a new kind, unexpected when the original action (1.1) of the $\mathrm{Y}_{2}$ models is considered perturbatively. The situation is similar to that found in [20, 21] and we suggest that also in the case of the $\mathrm{Y}_{2}$ models there might be "new universality classes which cannot be seen in conventional perturbation theory". 
The new universality classes arising along the lines $X$ could perhaps be also described by other actions, different from (1.1), such that their physical content is more transparent. A possible candidate would be the $\mathrm{GN}_{2}$ model and an additional scalar field theory in the high temperature phase, with both sectors coupled to each other in such a way that the spectrum of each sector does not change much. As an example one could imagine the coupling of the form: $\bar{\psi} F(\varphi) \psi$ with $F(\varphi)$ vanishing faster than $\varphi$.

Unfortunately, we see little chance to investigate these issues numerically. They constitute a part of the cross-over between two distinctly different universality classes. But the crossover phenomena are notoriously difficult on finite lattices, as one has to work very close to the critical point in order to disentangle various mixed scaling tendencies. The difficulties we have encountered at $\lambda=\infty$ and $\kappa=0.2$ suggest that there we are already too close to the crossover to be able to apply the simple MF method at those modest correlation lengths we could afford.

\section{Discussion and conclusion}

We presented some numerical evidence that the $\mathrm{Y}_{2}$ models belong to the same universality class as the $\mathrm{GN}_{2}$ models with the same chiral symmetry and $N_{F}$ even if a strong scalar self-interaction prohibits the use of the $1 / N_{F}$ expansion. Of course, the universality, or equivalence, of field theoretical models requires the equality of all observables like critical exponents, mass ratios, etc., in the continuum limit. Thus our evidence, based mainly on the scaling behaviour of the fermion mass, might seem to be only fragmentary. However, the significance by our results is amplified by the agreement with the MF method. This method makes predictions of the same type as the $1 / N_{F}$ expansion in the lowest order, but has been found to be applicable in a much wider range of parameters. The MF method thus seems to be the suitable analytic method for studying the $\mathrm{Y}_{2}$ models, provided the pure scalar sector is taken into account fully, which is not difficult numerically [10, 18]. The universality is a straightforward prediction of this approach.

We found that the scaling law (1.2) also holds when the bare kinetic term of the scalar field is negative. For $\kappa<0$ the MF approximation works even better than for $\kappa>0$. We think that this is due to a larger distance from the cross-over between the $\mathrm{GN}_{2}$ and the spin model universality classes. As the bare scalar field in $2 \mathrm{D}$ is dimensionless, the $\mathrm{Y}_{2}$ models can be chosen with quite arbitrary scalar field self-couplings and the $\varphi^{4}$ term, used in eq. (1.1), is only an example. However, we believe that our investigation of the $\mathrm{Y}_{2}$ models (2.1) with $\lambda=\infty$, i.e. $|\phi|=1$ is generic for a broad class of the $\mathrm{Y}_{2}$ models with quite arbitrary scalar self-couplings.

All that leads us to the conclusion that the $\mathrm{Y}_{2}$ models belong to the same universality class as the $\mathrm{GN}_{2}$ models with the same chiral symmetry and $N_{F}$ if $\kappa<\kappa_{c}$. Here $\kappa_{c}$ is the boundary of the high temperature phase of the pure scalar model with some rather general self-couplings. The $\mathrm{GN}_{2}$ models seem to be the most economic representatives of these universality classes, as they do not contain any ballast of irrelevant parameters.

However, this conclusion does not imply that the $\mathrm{Y}_{2}$ models are completely superfluous. It could be that the continuum limits taken at $\kappa=\kappa_{c}$ could lead to new universality classes, as some our speculations indicate. 


\section{A Appendix}

As mentioned in section 6 one can imagine that the ratio $\alpha=m_{\phi} / m_{F}$ can be tuned when one approaches the critical line $\kappa=\kappa_{c}(\lambda), y=0$. The value of $\alpha$ depends on the trajectory in the bare parameter space along which the continuum limit is approached. Let us consider a trajectory of the type $\mathrm{X}$ in figure 10, where $\kappa_{c}(\lambda)$ is approached from below.

In the $\mathrm{U}(1)$ case at $\lambda=\infty$ the limit $y=0$ is the well-known XY model, with the scalar mass vanishing near $\kappa=\kappa_{c}$ and the magnetic susceptibility diverging as:

$$
\chi \sim 1 / m_{\phi}^{2-\eta} \sim \exp \left[b\left(\kappa_{c}-\kappa\right)^{-\nu}\right], \quad \nu=\frac{1}{2}, \quad \eta=\frac{1}{4} .
$$

The approach to $\kappa=\kappa_{c}(\lambda), \lambda=\infty$ at fixed ratio $\alpha=m_{\phi} / m_{F}$ is described by a function $y(\kappa)$. According to the MF prediction (1.2) the fermion mass is given by:

$$
a m_{F} \approx \mu_{F} \exp \left(-\frac{1}{2 \beta_{0}} \frac{1}{\chi y^{2}}\right) .
$$

The ratio $\alpha$ now reads:

$$
\alpha \approx \frac{m_{\phi}}{\mu_{F}} \exp \left(\frac{1}{2 \beta_{0}} \frac{1}{\chi y^{2}}\right)
$$

This can be solved for $y$ :

$$
y^{2}(\kappa) \approx \frac{1}{2 \beta_{0}\left(\log \left(\alpha \mu_{F}\right)-\log m_{\phi}\right)} \cdot \frac{1}{\chi}
$$

where the dependence of $\chi$ and $m_{\phi}$ on $\kappa$ is given by (A.1). This formula can be used only as a rough approximation for the trajectory. On can expect it to be applicable at most for large $\alpha$ and not very close to $\kappa=\kappa_{c}$ (but close enough to apply (A.1)). Under these conditions eq. (A.4) can be simplified by keeping only the leading exponential dependence on $\left(\kappa_{c}-\kappa\right)$ in the r.h.s.:

$$
y^{2}(\kappa) \sim \frac{1}{\log \alpha} \exp \left[-b\left(\kappa_{c}-\kappa\right)^{-\nu}\right] .
$$

\section{References}

[1] D.J. Gross and A. Neveu, Phys. Rev. D10 (1974) 3235.

[2] E. Witten, Nucl. Phys. B145 (1978) 110.

[3] D. Lurié and A.J. Macfarlane, Phys. Rev. 136 (1964) 876;

T. Eguchi, Phys. Rev. D17 (1978) 611;

D. Campbell, F. Cooper, G.S. Guralnik and N. Snyderman, Phys. Rev. D19 (1979) 549;

K. Shizuya, Phys. Rev. D21 (1980) 2327.

[4] Y. Nambu, in Proceedings of 1989 Workshop on Dynamical Symmetry Breaking, p. 1, eds. T. Muta and K. Yamawaki (Nagoya University, Nagoya, 1990);

V.A. Miranski, M. Tanabashi and K. Yamawaki, Phys. Lett. B221 (1989) 177; Mod. Phys. Lett. A4 (1989) 1043;

W. Bardeen, C. Hill and M. Lindner, Phys. Rev. D41 (1990) 1647;

W. Suzuki, Phys. Rev. D41 (1990) 3457; Mod. Phys. Lett. A5 (1990) 1205.

[5] J. Shigemitsu, Phys. Lett. B226 (1989) 364. 
[6] A. Hasenfratz, P. Hasenfratz, K. Jansen, J. Kuti and Y. Shen, Nucl. Phys. B365 (1991) 79.

[7] J. Zinn-Justin, Nucl. Phys. B367 (1991) 105.

[8] K. Tamvakis and G.S. Guralnik, Nucl. Phys. B146 (1978) 224.

[9] A.K. De, E. Focht, W. Franzki and J. Jersák, Nucl. Phys. B (Proc. Suppl.) 30 (1993) 662.

[10] A.K. De, E. Focht, W. Franzki, J. Jersák and M.A. Stephanov, Phys. Lett. B 308 (1993) 327.

[11] E. Focht, Nucl. Phys. B (Proc. Suppl.) Proceedings of the XI Int. Symposium on Lattice Field Theory, Dallas 1993 (to be published).

[12] Y. Shen, Phys. Lett. 304B (1993) 295.

[13] R.F. Dashen, B. Hasslacher and A. Neveu, Phys. Rev. D12 (1975) 2443.

[14] S.-S. Shei, Phys. Rev. D 14 (1976) 535.

[15] P. Forgács, F. Niedermayer and P. Weisz, Nucl. Phys. B367 (1991) 123; Nucl. Phys. B367 (1991) 144;

P. Forgács, S. Naik and F. Niedermayer, Phys. Lett. B283 (1992) 282.

[16] I.K. Affleck, Phys. Lett. 109B (1982) 307;

Y. Cohen, S. Elitzur and E. Rabinovici, Phys. Lett. 104B (1981) 289; Nucl. Phys. B220 (1983) 102 .

[17] W. Bock, A.K. De, C. Frick, J. Jersák and T. Trappenberg, Nucl. Phys. B378 (1992) 652 ;

A.K. De and J. Jersák, in Heavy Flavours, eds. A. Buras and M. Lindner (World Scientific, Singapore, 1992) p.732.

[18] M. Stephanov, Nucl. Phys. B (Proc. Suppl.) Proceedings of the XI Int. Symposium on Lattice Field Theory, Dallas 1993 (to be published).

[19] N. Attig, L. Bélanger, R. Lacaze, A. Morel, B. Petersson and M. Wolff, Nucl. Phys. B340 (1990) 245.

[20] S. Caracciolo, R.G. Edwards, A. Pelissetto and A.D. Sokal, preprint IFUP-TH-3493, hep-lat 9307022.

[21] A. Sokal, Nucl. Phys. B (Proc. Suppl.) Proceedings of the XI Int. Symposium on Lattice Field Theory, Dallas 1993 (to be published). 